\section{(A) Check for updates}

Cite this: Analyst, 2021, 146, 5880

\title{
Exploring AdaBoost and Random Forests machine learning approaches for infrared pathology on unbalanced data sets $\uparrow$ :
}

\begin{abstract}
Jiayi Tang, (D) Alex Henderson (D) * and Peter Gardner (D)
The use of infrared spectroscopy to augment decision-making in histopathology is a promising direction for the diagnosis of many disease types. Hyperspectral images of healthy and diseased tissue, generated by infrared spectroscopy, are used to build chemometric models that can provide objective metrics of disease state. It is important to build robust and stable models to provide confidence to the end user. The data used to develop such models can have a variety of characteristics which can pose problems to many model-building approaches. Here we have compared the performance of two machine learning algorithms - AdaBoost and Random Forests - on a variety of non-uniform data sets. Using samples of breast cancer tissue, we devised a range of training data capable of describing the problem space. Models were constructed from these training sets and their characteristics compared. In terms of separating infrared spectra of cancerous epithelium tissue from normal-associated tissue on the tissue microarray, both AdaBoost and Random Forests algorithms were shown to give excellent classification performance (over 95\% accuracy) in this study. AdaBoost models were more robust when datasets with large imbalance were provided. The outcomes of this work are a measure of classification accuracy as a function of training data available, and a clear recommendation for choice of machine learning approach.
\end{abstract}

Received 30th October 2020 Accepted 10th May 2021

DOI: 10.1039/d0an02155e

rsc.li/analyst human tissue is composed of essentially the same chemical species, advanced chemometric methods are required to classify the composite tissue types present; in particular, those that exhibit diseased characteristics. When attempting to develop a chemometric model, care must be taken to ensure its stability under different performance conditions. Typically, exemplar tissue samples will be examined by a trained pathologist and analysed using infrared spectroscopy. The pathologist will indicate regions of interest, while the spectroscopist identifies these regions in the data, before submitting them to the model-building process. The tissue under examination can have varying degrees of cell type, both in terms of naturally occurring diversity and those cells modified by the disease under investigation. Therefore, the data collected can have a variety of composition which presents an additional problem in the model-building process. In this paper we explore the influence of this composition using two machine learning algorithms.

\section{Machine learning}

Machine learning (ML) is a branch of artificial intelligence. It allows a computer to learn from data and to improve decision making with experience. ML refines a model that can be used to predict outcomes of inquiry, based on previous learning. There are two types of machine learning: supervised and unsu- 
pervised. For supervised learning, a labelled set of inputoutput pairs is provided to the algorithm which then learns a model which can reproduce this mapping. ${ }^{20}$ Unsupervised learning can be described as knowledge discovery. Here, the objective is to find hidden patterns in data. ${ }^{20}$ There are no defined answers, as there is no specific existing pattern to find. ${ }^{20,21}$

In the field of spectroscopy, a number of studies have combined FTIR hyperspectral imaging data with the Random Forests $^{\mathrm{TM}}$ classification algorithm. Leslie et al. applied it on lymph node histopathology in $2015,{ }^{22}$ Mittal et al. used Random Forests on a four-class classification for digital breast histopathology ${ }^{17}$ and simultaneous cancer and tumour microenvironment detection. ${ }^{23}$ Pilling et al. also showed that for biopsy tissue mounted on glass substrates, Random Forests could give classification accuracies over $95 \%{ }^{4}$

Random Forests. The Random Forests ${ }^{\mathrm{TM}}$ algorithm is a supervised machine learning technique based on an array of decision trees (Fig. 1). Random Forests is one of many ensemble methods, that construct a group of classifiers and then sort previously unseen data by taking a vote of predictions made by the set of weak learners; in this case decision trees. ${ }^{24}$ This type of ensemble approach is termed bagging. Ensemble methods are well established as a way of obtaining a highly accurate classifier by combining many less accurate ones. ${ }^{24}$ In the case of Random Forests, the weak classifier is a decision tree. During the model building process, the Random Forests algorithm creates many decision trees, as required, each with a different sub-sample of the available variables. Each decision tree will develop its own route to classification. The trees are then tested using unseen data and the outcome of each tree is recorded. A majority vote amongst all the trees in the forest is taken and the overall model 'votes' on the outcome. ${ }^{25}$

AdaBoost. Random Forests has generally been the preferred machine learning method in the bio-spectroscopy field. However, alternative ensemble methods have yet to be explored. One such ensemble approach, boosting, again uses a weak learner internally, but here uses a triplet of learners, each with a different, but related input. Boosting can be used iteratively to improve classification performance. The most well-known boosting method is the Adaptive Boosting (AdaBoost) algorithm of Freund and Schapire. ${ }^{26,27}$ AdaBoost was the first practical boosting algorithm and remains one of the most widely used and studied, with applications in numerous fields. ${ }^{28}$ The AdaBoost approach involves a user defined number of iterations. In each iteration a triplet of weak learners is applied to the entire training set and the outcome compared to the expected sample labelling. An error function is then determined which provides a weighting applied to each spectrum for the next iteration. This has the effect of downweighting spectra that were correctly classified in the previous iteration and up-weighting those that were misclassified. Subsequent iterations will then focus on spectra that have yet to be correctly classified. To determine the predicted class of a previously unseen test subject, each of the weighted weak learners, from each iteration, are provided with the unseen data and a weighted majority vote is taken on their decision. ${ }^{29}$ This is shown schematically in Fig. 2.

Adaboost is considered more effective at handling an unbalanced dataset than Random Forests, since the minority class, which is much more likely to be misclassified, can be given higher weighting in subsequent iterations, and can improve
A)

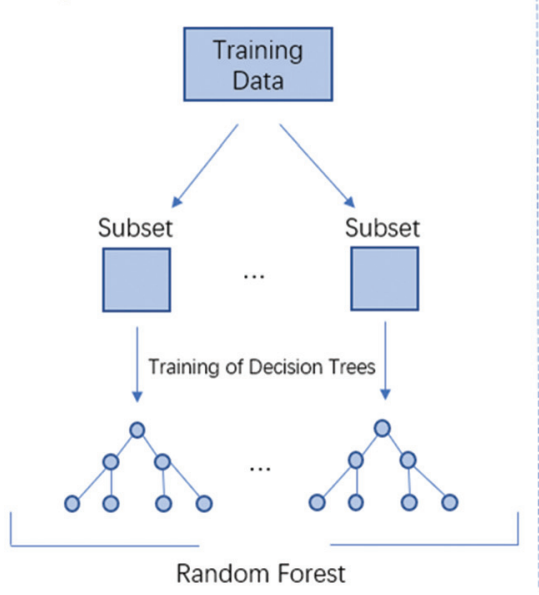

B)

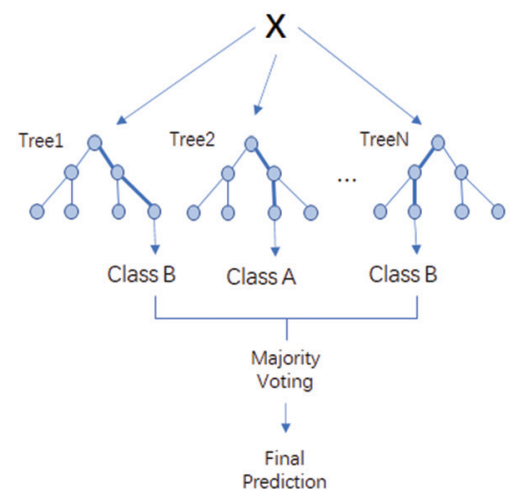

C)
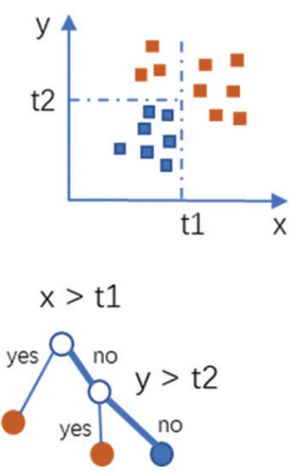

Fig. 1 Simplified Random Forests method. (A) Features in the training data are divided into multiple subsets and used to train individual decision trees in the forest. (B) When an unknown input $X$ is introduced to the trained forest, each tree will make its own prediction. The final prediction is decided by majority voting of all trees. (C) An example of how a decision tree makes a prediction, where blue squares represent samples in class $A$ while orange squares represent samples in class $\mathrm{B}$, and $\mathrm{t} 1$ and $\mathrm{t} 2$ are two example features used at nodes. 

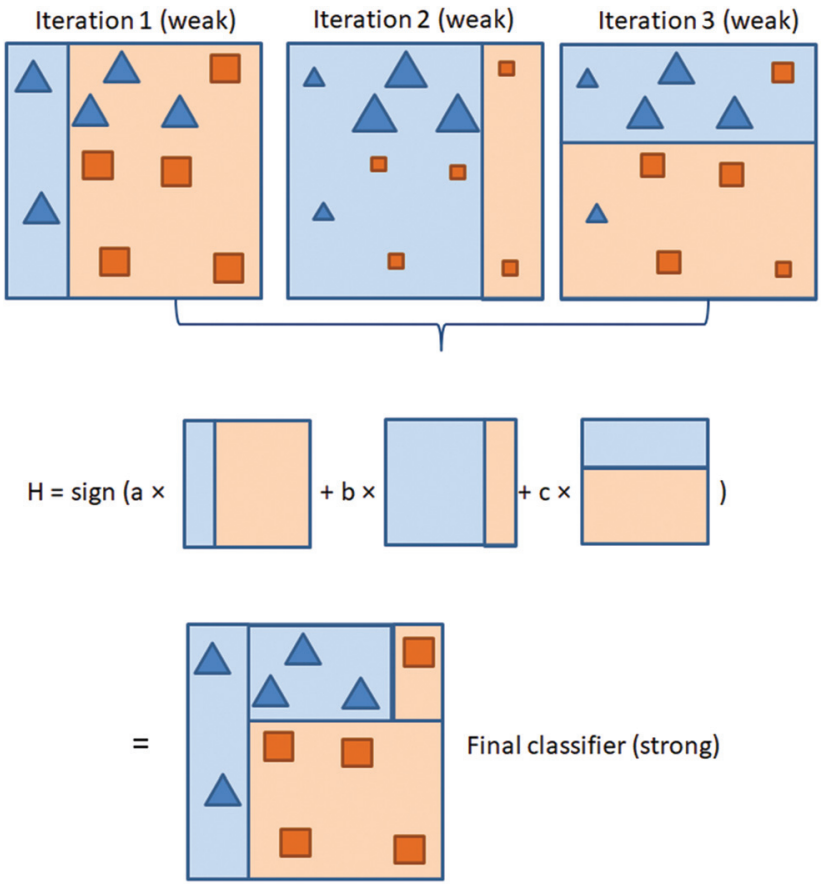

Fig. 2 A schematic of the AdaBoost process. Blue triangles and orange squares represent features, with the size of the features representing weighting. Iteration 1 , all features carry equal weight. Iteration 2 , correctly classified features (in iteration 1) down-weighted, incorrectly classified feature up-weighted. Iteration 3, correctly classified features (in iteration 2) down-weighted, incorrectly classified feature upweighted. The combination of the iterations produces a final classifier that is strong.

the performance of weak learners regardless of whether training data is balanced or unbalanced. ${ }^{30}$

\section{Unbalanced data}

In the real world, it is highly unlikely that there will be the same number of spectra of each class in any training data set, without specific pre-processing to that effect. Biased results can be produced if directly applying classification to these data. ${ }^{31,32}$ Studies, using a number of different approaches, have been conducted in various fields, to reduce the influence of unbalanced data sets in model predictions..$^{30,33-35}$

In terms of clinically related studies, data encountered for classification is often unbalanced. In the case of cancer biopsy samples, the class of interest, possibly dysplastic or cancerous epithelial cells, may be small compared with the total number of cells within the tissue sample which may consist of predominately stroma. Therefore, application of hyperspectral image analysis, where the pixels are arranged in a grid pattern, will result in the number of spectra from each cell type (class) being unequal. ${ }^{36}$

If a classification model is established to separate epithelium cells from stroma, a very unbalanced dataset will be obtained, which is inherently biased. When the number of samples in one class (majority) largely exceeds the number of samples in the other (minority), data mining algorithms tend to favour the majority class. The minority class, which is frequently the class of interest (positive class), can have poor classification accuracy due to the biased model. Therefore, techniques are required to ensure that a model can efficiently identify minority classes.

Different methods, for example weighting, can be employed to alleviate bias. Here, we explore re-sampling methods. ${ }^{37}$ There are two commonly used re-sampling methods: undersampling and over-sampling. The class distribution can be balanced by either duplicating selected members of the minority class (over-sampling), or removing selected examples from the majority class (under-sampling). ${ }^{36}$ Under-sampling. and over-sampling can be performed in different ways. ${ }^{30}$ Random under-sampling balances two classes by randomly removing data from the majority class to match the number of samples in the minority class. Random over-sampling replicates samples in the minority class until the number of samples matches that of the majority class.

In this work we compare the classification accuracy of models developed using two machine learning techniquesAdaBoost and Random Forests- - to data from infrared spectroscopic analysis of human breast tissue biopsies, where that data has unbalanced class structure, and also when underand over-sampling strategies have been employed to mitigate this.

\section{Methodology}

\section{The sample}

A formalin fixed, paraffin embedded, breast tissue microarray (TMA) - ID BR20832 - was used for this study (US Biomax, Rockville, MD, USA). The human tissue was collected under approved HIPPA protocols and approved for commercial product development. The TMA contained 15 pathologically indicated non-malignant cores and 192 malignant cores: in total 207 breast tissue biopsy cores, each $1 \mathrm{~mm}$ in diameter. Each core was biopsied from a different patient. A $5 \mu \mathrm{m}$ thick section was floated onto a standard histology glass slide and stained using haematoxylin and eosin (H\&E). An adjacent section of the same thickness was floated onto a $\mathrm{BaF}_{2}$ slide for infrared spectroscopic analysis. This IR sample was not dewaxed, reducing the likelihood of inducing chemical changes during deparaffinization, and decreasing spectral Mie scattering due to the closer refractive index values between paraffin and sample. ${ }^{38}$

Fifty cores were selected from the TMA, which included forty cores with stage II breast cancer and ten normal-associated breast tissue cores: histological normal tissue adjacent to the tumor (NAT) from non-malignant cores. ${ }^{39}$

\section{Data acquisition}

FTIR scans were obtained in transmission mode using an Agilent Cary 670-IR spectrometer fitted with a liquid nitrogencooled $128 \times 128$ focal plane array (FPA), mercury cadmium telluride (MCT) detector. An Agilent Cary 620-IR imaging 
microscope, with a $\times 15$ Cassegrain objective, was coupled to the spectrometer. The instrument produced a resultant fieldof-view of $704 \times 704 \mu \mathrm{m}$, with a corresponding pixel size of $5.5 \mu \mathrm{m}$.

The FTIR instrument is fitted with a sealable enclosure, surrounding the sample stage and optics, through which dry air is continuously delivered. The relative humidity within this chamber was reduced to zero percent prior to any data acquisition. This has the benefit of removing any water vapour that might be otherwise present in the optical path and subsequently recorded as part of the sample's spectrum. Before imaging, background scans were taken from a region, selected to be clean and paraffin free, in the form of a single FPA tile with 128 co-added scans at a spectral resolution of $5 \mathrm{~cm}^{-1}$. For tissue analysis, 96 co-added sample scans were measured. Chemical images of each core were acquired as a $2 \times 2$ mosaic; each mosaic taking approximately 15 minutes to collect. Interferograms were processed using Happ-Genzel apodisation with two levels of zero filling and a spectral range of 900 to $3800 \mathrm{~cm}^{-1}$.

\section{Data pre-processing}

All data were pre-processed using MATLAB® R2017a (The MathWorks Inc., Natick, MA, USA). Infrared spectra for each biopsy core were extracted from the mosaic as a $256 \times 256 \times$ 1478 hypercube, where each hypercube consisted of 65536 spectra, each with 1478 data points.

FTIR chemical images of each of the breast tissue cores were generated and compared to the H\&E stained sections. Fig. 3 shows examples of both H\&E stained and infrared hyper-

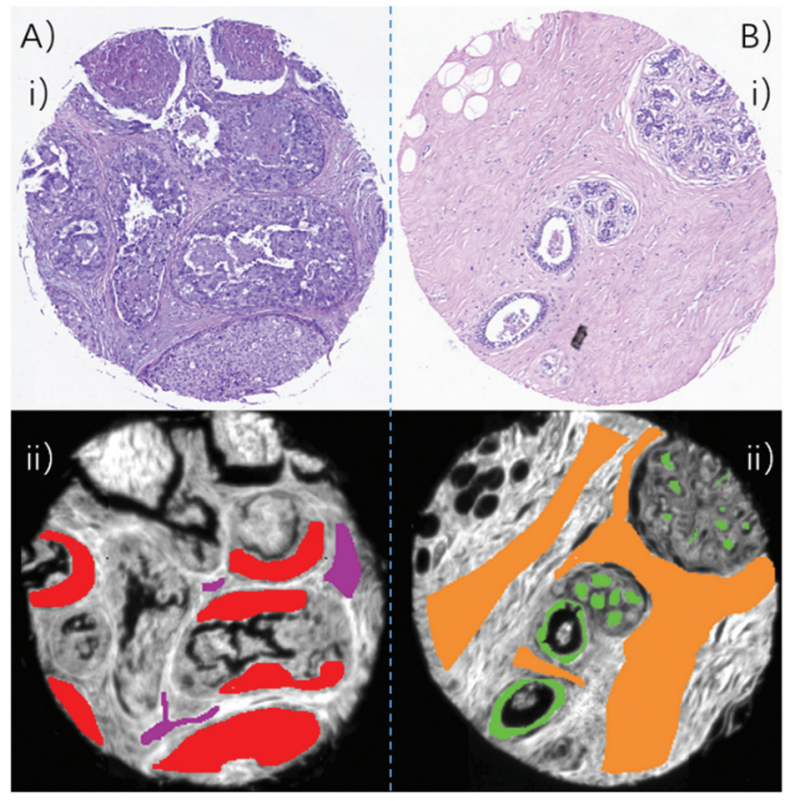

Fig. 3 (A) Sample image of cancerous core (i) bright field H\&E image and (ii) annotated infrared image. (B) Sample image of NAT core (i) bright field H\&E image and (ii) annotated infrared image, where red indicates cancerous epithelium, purple indicates cancerous stroma, green indicates NAT epithelium and orange indicates NAT stroma. spectral images of a cancerous core, and a core containing normal associated tissue. Examples of the manually annotated regions, from which spectra were extracted, are indicated on the infrared images. Regions of epithelium were identified according to World Health Organisation (WHO) documentation: WHO Classification of Tumours in the Breast. ${ }^{40}$

Principal components-based noise reduction was used to improve the signal-to-noise ratio of raw spectra from each annotated area; the first 80 principal components being retained. Spectra were quality tested to remove data obtained from areas with little or no tissue, based on the intensity of the amide I band; spectra having absorbance between 0.1 and 2 being retained. Spectral regions describing the absorption bands of paraffin wax were removed with spectral ranges 1000 to $1319 \mathrm{~cm}^{-1}, 1481$ to $1769 \mathrm{~cm}^{-1}$, and 2986 to $3569 \mathrm{~cm}^{-1}$ being retained for further processing. Each spectrum was then converted to its first derivative using the Savitzky-Golay algorithm, based on a fourth order polynomial, with a window size of 19 data points. Further spectral ranges were then deleted from the derivatized data to remove the end regions, which can be influenced by the derivatization process, to leave a data set comprising the spectral ranges: 1019-1300, 1500-1750 and 3005-3550 $\mathrm{cm}^{-1}$. No spectral normalisation was performed since all tissue samples were part of the same tissue microarray, and thus have the same thickness.

\section{Training and test sets}

The data was separated into two collections: training data and independent test data. The training data contained 32 cancerous and 8 normal-associated cores, 40 in total, while the independent test data contained 8 cancerous and 2 normal-associated cores, 10 in total. Each core originated from a different patient. To eliminate the effect of different size of annotation areas contributing differently to each other in the model, the same number of pixels (327) were randomly selected from each core to match with the minimum number of pixels in the 40 cores, and maximising the use of different cores from different patients.

Independent test set. From the TMA we identified eight cores as being cancerous tissue and two cores with normalassociated tissue (NAT). Spectra from annotated regions in these cores were extracted and used to form an independent test set. This independent test set was used for all model assessment procedures and comprised 1352 cancer-related spectra and 338 normal-associated tissue spectra: 1690 spectra in total. These ten cores were removed from that analysis pool to prevent crossover between model building and model assessment. Recall that each core is from a separate patient.

Training sets A: same overall training set size, unbalanced class sizes, unique spectra. Five training sets were generated from the pool of training data using the process shown in Fig. 4. We randomly selected 2500 spectra of cancerous tissue and 2500 spectra of normal-associated tissue from our collection of annotated spectra. We removed 500 spectra from the normal-associated pool, and randomly selected an additional 500 cancerous spectra from the annotated collection. 


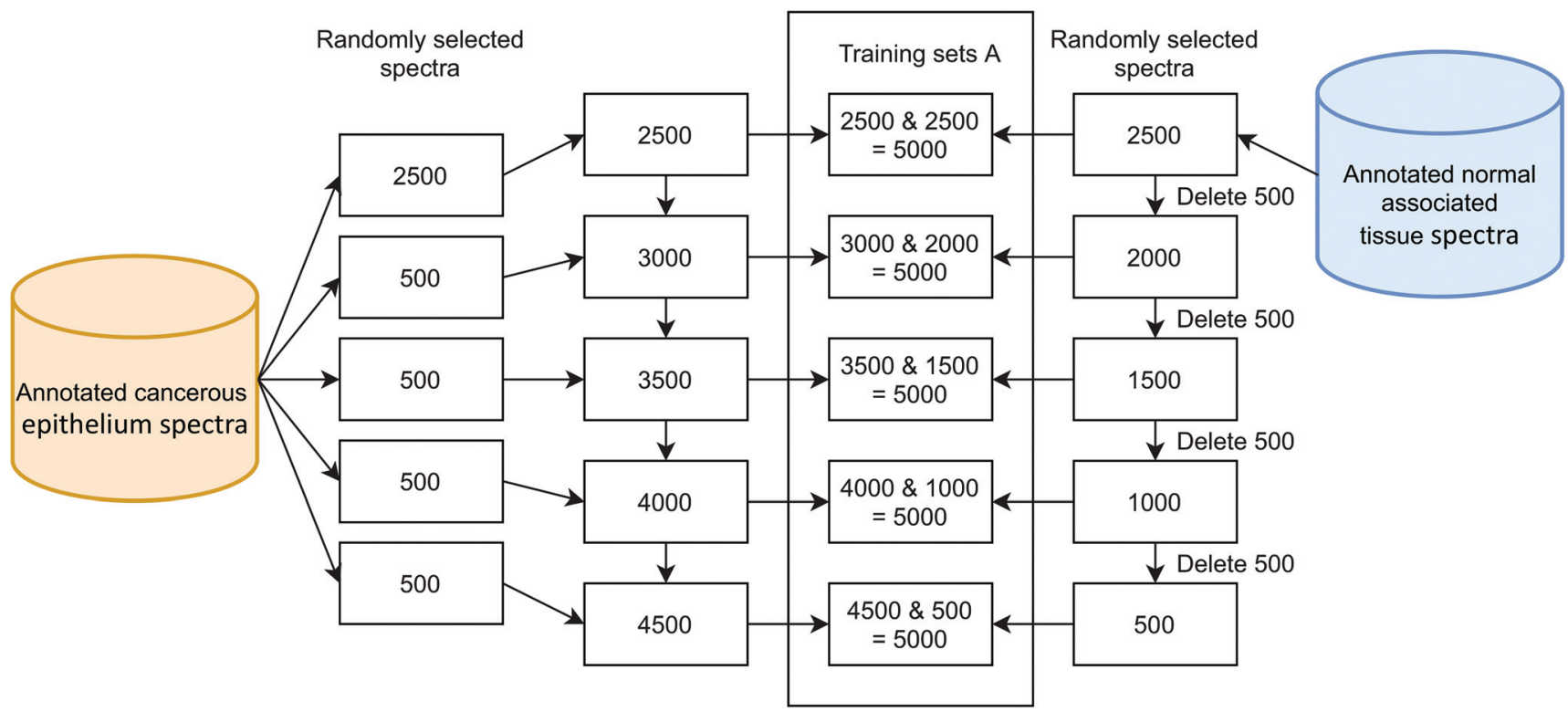

Fig. 4 Schematic showing the process involved in generating training sets A, with equal overall size, but an imbalance in composition. All spectra are unique.

Combining these produced a training set with 5000 spectra, but instead of there being 2500 of each type, there were 3000 cancerous spectra and 2000 normal-associated spectra. A further three training sets were generated, in each case adding new cancerous spectra, while removing normal-associated spectra, as shown in Fig. 4 and Table 1.

Training sets B: different overall training set size, balanced class sizes (under-sampled), unique spectra. Starting in the same manner as for training sets A, we randomly selected 2500 spectra of cancerous tissue and 2500 spectra of normal-associated tissue. These two sets of spectra were combined to form a balanced training set with 5000 spectra. To simulate scenarios in which there are a limited supply of spectra in the minority class, we reduced the number of normal-associated tissue spectra by randomly removing 500 spectra. The majority class, cancer, was then under-sampled by randomly removing 500 spectra to produce an equal number of spectra (2000) in both the majority (cancer) and minority (NAT) classes. These were

Table 1 Composition of training sets A: unbalanced classes of same overall size and unique composition. Rows indicate the composition of each training set, with coloured squares indicating the number of cancer spectra (orange) and normal-associated tissue spectra (blue)

\begin{tabular}{lllrl}
\hline $\begin{array}{l}\text { Initial } \\
\text { ratio }\end{array}$ & $\begin{array}{l}\text { Num } \\
\text { cancer }\end{array}$ & Training sets A & $\begin{array}{l}\text { Num } \\
\text { NAT }\end{array}$ & Total \\
\hline $50: 50$ & 2500 & & 2500 & 5000 \\
$60: 40$ & 3000 & & 2000 & 5000 \\
$70: 30$ & 3500 & 1500 & 5000 \\
$80: 20$ & 4000 & 1000 & 5000 \\
$90: 10$ & 4500 & & 500 & 5000
\end{tabular}

Table 2 Composition of training sets B: balanced classes of different total size, but unique composition, generated by under-sampling the majority class to match the number in the minority class. Rows indicate the composition of each training set, with coloured squares indicating the number of cancer spectra (orange) and normal-associated tissue spectra (blue)

\begin{tabular}{|c|c|c|c|c|}
\hline $\begin{array}{l}\text { Initial } \\
\text { ratio }\end{array}$ & $\begin{array}{l}\text { Num } \\
\text { cancer }\end{array}$ & Training sets B & $\begin{array}{l}\text { Num } \\
\text { NAT }\end{array}$ & Total \\
\hline $50: 50$ & 2500 & & 2500 & 5000 \\
\hline $60: 40$ & 2000 & & 2000 & 4000 \\
\hline $70: 30$ & 1500 & & 1500 & 3000 \\
\hline $80: 20$ & 1000 & & 1000 & 2000 \\
\hline $90: 10$ & 500 & & 500 & 1000 \\
\hline
\end{tabular}

combined to generate a smaller, but equally balanced training set with 4000 spectra. This under-sampling was repeated a further three times to produce balanced training sets with 3000 , 2000 and 1000 spectra in total (Fig. 5). These training sets contain unique spectra, and their composition is shown in Table 2.

Training sets C: different overall training set size, balanced class sizes (over-sampled), minority class duplicated. Over-sampling is the addition of examples into the minority class. There are a variety of approaches to this, including:

1. Determine the difference in the size between the majority class and minority class. Replicate each of the spectra in the minority class enough times to match this difference. Append the replicates to the minority class. 


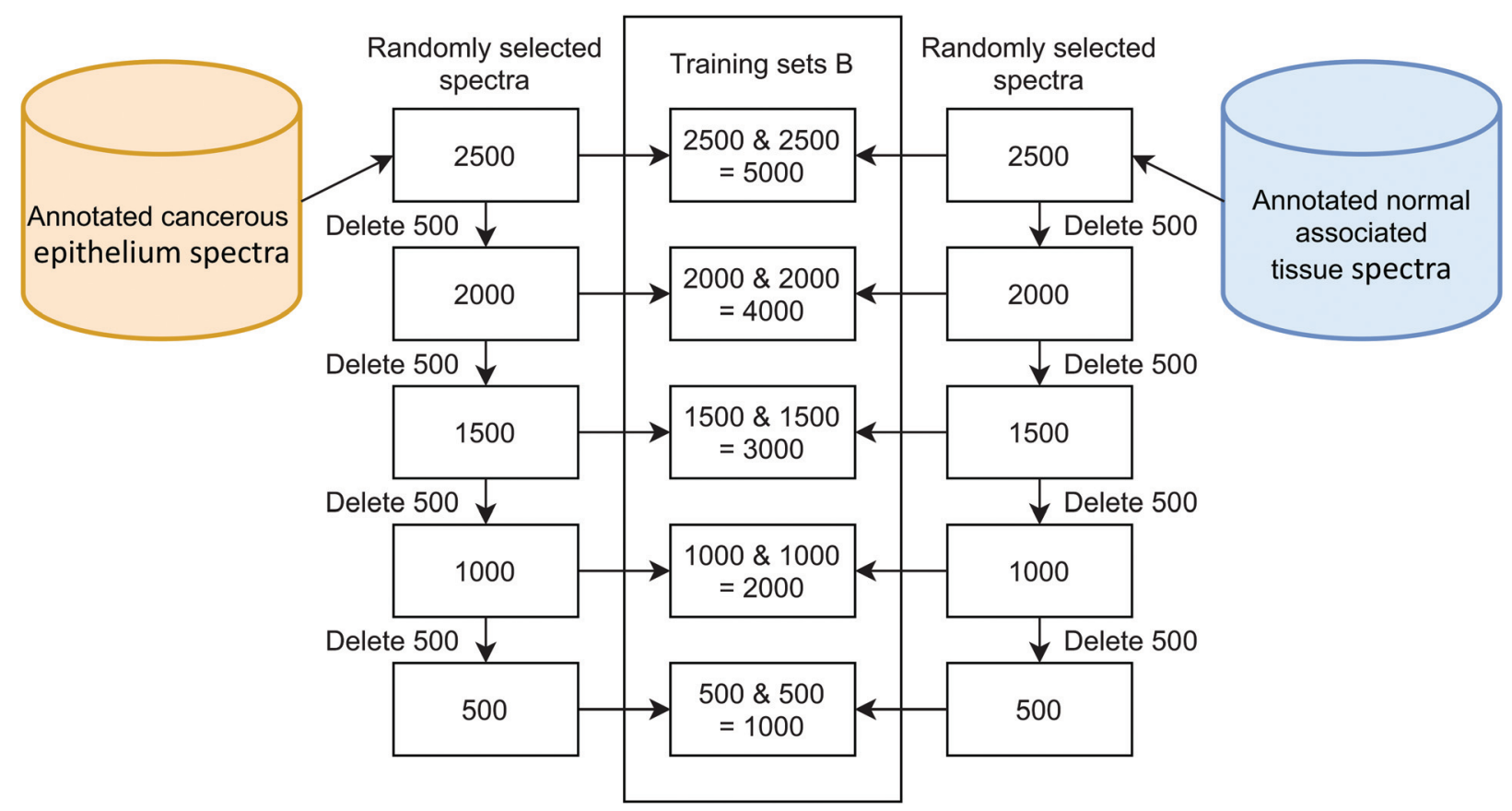

Fig. 5 Schematic showing the process used to generate training sets B, with equal class size, but different total number of unique spectra.

2. Randomly select spectra from the minority class until the total equals the size of the majority class. This is sampling with replacement.

3. Determine the difference in size between the majority class and minority class. Randomly select spectra from the minority class enough times to match this difference. Append the replicates to the minority class.

4. Perform an interpolation of one or more spectra in the minority class, and append these to that class, to increase the number of spectra available.

The first approach allows for an (almost) equal number of each minority spectrum to be present in the over-sampled training data. The fully random nature of the second approach means that not all spectra in the original minority class may be included in the over-sampled set. There will be no guarantee of the degree of duplication of each minority class spectrum. The possibility that any of the original minority may be missing in the outcome means information is being lost. The third approach is a modification of the second. Here the entire minority class is included in the outcome, with the remainder being topped up randomly. No information is lost. The fourth approach does not duplicate spectra exactly; rather it creates interpolated versions of minority class spectra. This is expanded upon in the paper by Blagus and Lusa ${ }^{41}$ where they describe their Synthetic Minority Oversampling Technique (SMOTE) method.

In this study we used approach number three. This has the advantage of ensuring each member of the minority class is represented in the training set. Starting in the same manner as for training sets A and B, we randomly selected 2500 spectra of cancerous tissue and 2500 spectra of normal-associated tissue. These two sets of spectra were combined to form a balanced training set with 5000 spectra. The generation of the cancerous epithelium component of the training sets followed the same pattern as training sets A: For each set an additional 500 spectra were randomly selected from the pool of annotated spectra. To generate the subsequent NAT part of each training set, 500 spectra were removed from the first set, producing 2000 spectra. From these 2000 were randomly selected 1000 spectra which were appended to the 2000 to create a NAT training set of 3000 spectra. The 3000 cancerous spectra and 3000 NAT spectra were combined to produce a balanced training set of 6000 spectra.

Further training sets were generated in a similar manner. The cancerous component was topped up using previously unselected spectra from the overall pool. The NAT component was first reduced in number by deleting 500 . Then, the difference in size between the cancerous component and NAT component was calculated. The requisite number of spectra (this difference) was then randomly selected from the current, depleted NAT component. In this way, the heavily unbalanced classes, for example 4500 versus 500, had their NAT component topped up by sampling 4000 times from the pool that only contained 500 spectra (Fig. 6). This has the effect of creating a large degree of duplication in the NAT class, with no control over the distribution of that sampling. Indeed, the same spectrum could be added 4000 times (Table 3 ).

\section{Results}

The AdaBoost algorithm was employed to construct models from the various training sets developed above. The same data were then used to construct models using the Random Forests 


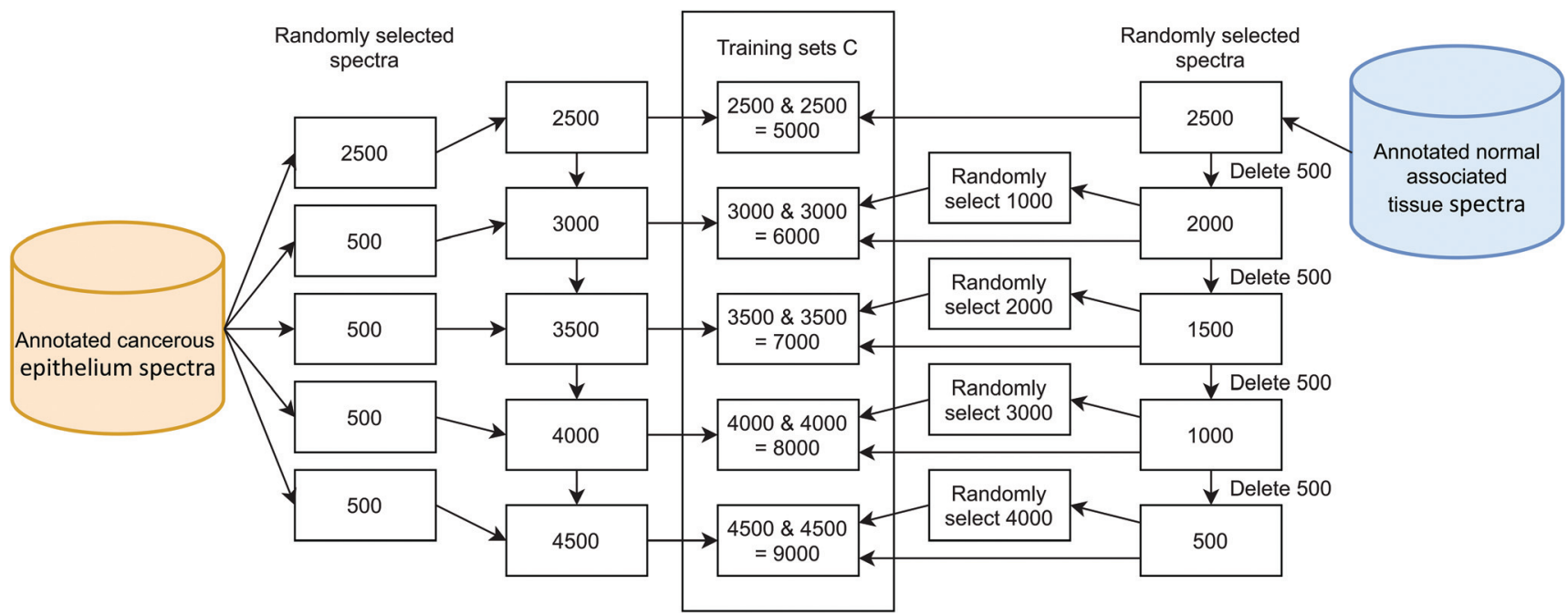

Fig. 6 Schematic showing the process used to generate training sets C, with equal class size, but different total number of spectra. Some normalassociated tissue spectra are unique while others are replicates, generated by over-sampling.

Table 3 Composition of training sets C: balanced classes of different total size generated by over-sampling. Rows indicate the composition of each training set, with coloured squares indicating the number of cancer spectra (orange) and normal-associated tissue spectra (blue). Cells labelled $U$ indicate unique spectra, while those labelled $\mathrm{D}$ indicate duplicates

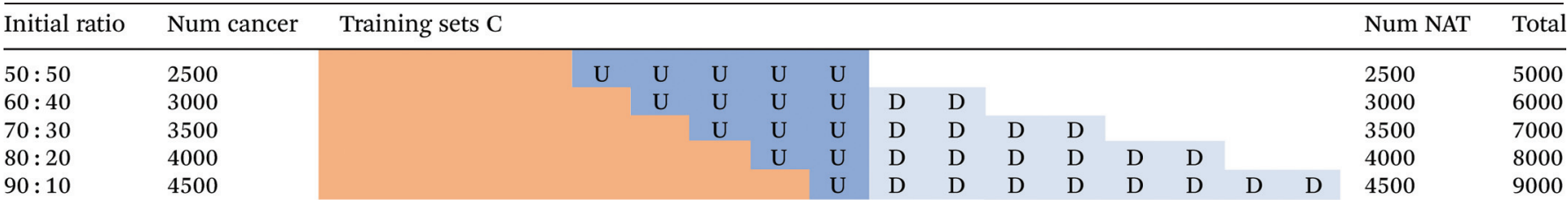

algorithm for comparison. All models were tested using the same independent test data set. In all cases each experiment was repeated five times to assess variability.

\section{AdaBoost results}

The AdaBoost.M1 algorithm, from the Statistics and Machine Learning Toolbox within MATLAB, was selected. AdaBoost.M1 is appropriate since only a two-class problem is considered here. 500 iterations were applied with a learning rate (to train an ensemble using shrinkage) equal to 1 .

Same overall training set size, unbalanced class sizes, unique spectra. Using Training sets A, we have training data with unbalanced ratios ranging from $50: 50$ to $90: 10$, cancer to normal-associated spectra respectively. AdaBoost models were constructed from these data, tested using the independent test set, and the results are shown in Fig. 7 . The median accuracy of each of the five repeats is also indicated on the plot, with the median being selected as a robust statistic in the presence of outliers.

Both cancer and NAT spectra are classified with over 95\% accuracy for the initial case of balanced classes containing 2500 spectra each. As the class imbalance grows the accuracy of the NAT class decreases while that of the cancer class grows until they cross between the $80: 20$ and $90: 10$ ratios, corresponding to $4000: 1000$ and $4500: 500$, cancer: NAT spectra respectively. Even at this final, large imbalance the classification accuracies are still over 94\%. This indicates that the AdaBoost method is robust to class imbalance.

Different overall training set size, balanced class sizes (under-sampled), unique spectra. Recall that the training data here (Training sets B) comprises an equal number of cancerous and normal-associated spectra, but with differing total number of spectra: 5000 to 1000. Therefore, each of these training data are balanced using the under-sampling methodology.

Fig. 8 shows the classification accuracy of each data set. The classification accuracy of the normal-associated spectra is approximately 99\% with 2500 spectra of each class and remains very high even when reduced to 500 spectra each. Conversely, the classification rate of cancerous spectra drops from $\sim 96 \%$ to $\sim 86 \%$ as the total number of spectra drop. This experiment was extended, reducing the numbers of spectra in each class much further. The results are shown in Fig. 9. When the number of spectra in each class is reduced below 100 , the classification rate of cancer spectra drops to zero and that of normal-associated tissue rises to $100 \%$. In both these 


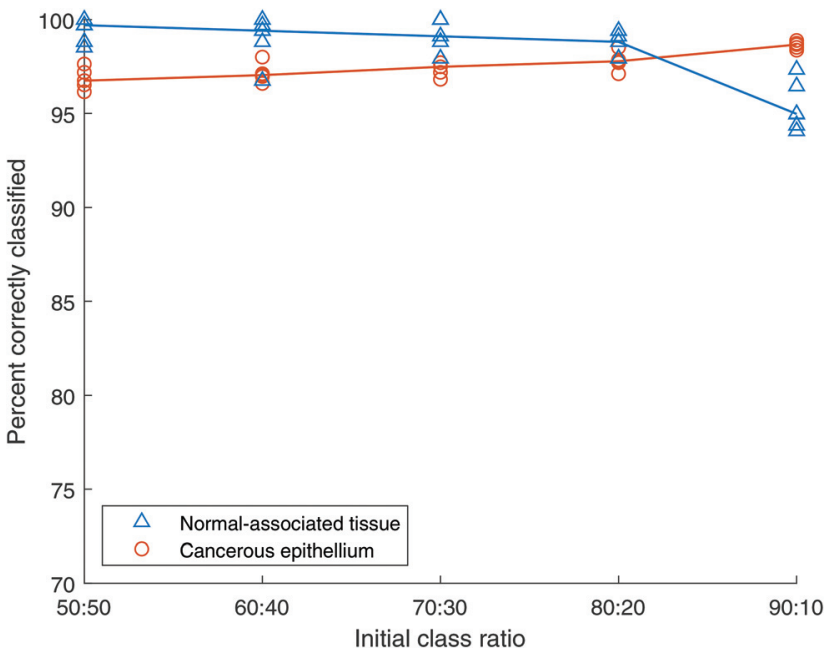

Fig. 7 Classification accuracy of AdaBoost with unbalanced training sets A. All spectra are unique. Blue triangles show normal-associated tissue (NAT) results, orange circles show cancer tissue results. The lines represent the median of NAT repeats in blue and cancer repeats in orange.

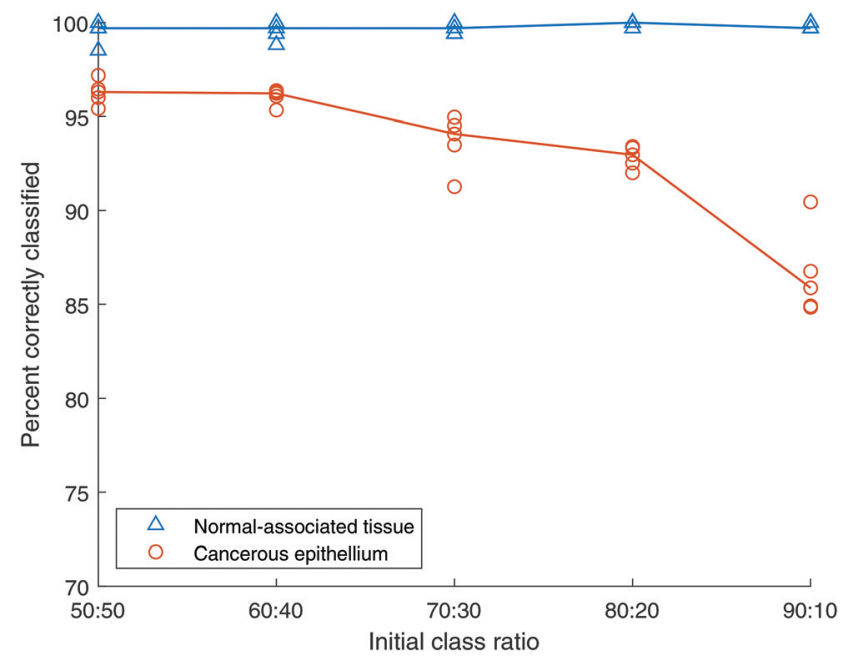

Fig. 8 AdaBoost classification accuracy using training sets B, with balanced classes of decreasing size, generated by under-sampling. All spectra are unique. Blue triangles show normal-associated tissue (NAT) results, orange circles show cancer tissue results. The lines represent the median of NAT repeats in blue and cancer repeats in orange.

cases, the classification rate of normal-associated tissue remains high, while that of cancer spectra falls, until the model fails completely. It appears that the normal-associated tissue spectra are being correctly classified, but some of the cancer spectra are also being classified as normal-associated. As the number of spectra decreases, this reaches a critical point where all spectra are classed as normal-associated and therefore all cancer spectra are misclassified.

Different overall training set size, balanced class sizes (oversampled), minority class duplicated. Generating balanced

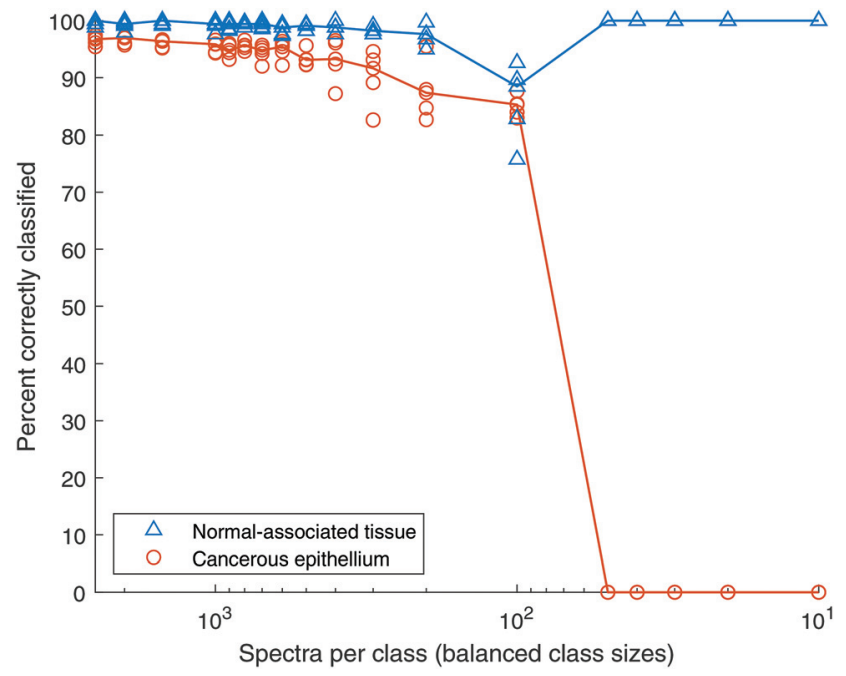

Fig. 9 Classification accuracy of AdaBoost with balanced classes of decreasing overall size. All spectra are unique. Blue triangles show normal-associated tissue (NAT) results, orange circles show cancer tissue results. The lines represent the median of NAT repeats in blue and cancer repeats in orange.

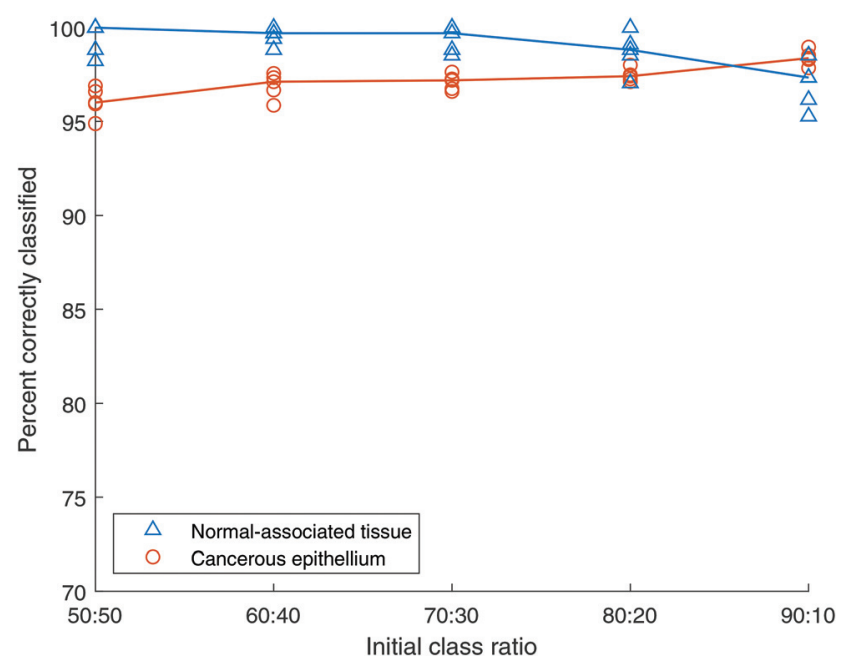

Fig. 10 AdaBoost classification accuracy with different unbalanced class ratios being balanced using the over-sampling approach. Training sets $C$, where the minority class contains duplicates. Blue triangles show normal-associated tissue (NAT) results, orange circles show cancer tissue results. The lines represent the median of NAT repeats in blue and cancer repeats in orange.

classes by under-sampling reduces the total number of spectra available to the learner. An alternative approach is oversampling. Here we explore the outcome of over-sampling on the classification rate of data with different initial class sizes.

Using Training sets $\mathrm{C}$ above, we duplicated normal-associated spectra to match the number of cancer spectra, for different initial class sizes. AdaBoost models were then constructed from these training sets and tested using the independent test set. The results are shown in Fig. 10. Here the $x$-axis 
shows the initial class ratio, prior to over-sampling. Each training set becomes balanced, but the number of spectra also increases. For example, for a scenario with an initial ratio of $50: 50$, the training set comprises 2500 of each class giving a total of 5000 spectra. Contrast this with an initial ratio of 90 : 10 which, following the oversampling exercise results in 9000 spectra.

The outcome here is similar to that produced by the unbalanced Training sets A, shown in Fig. 7, where the classification accuracy of the NAT class decreased slightly with increasing class imbalance, with the accuracy of the cancerous class increasing slightly. When the class sizes are balanced (50:50) the normal-associated spectra show a classification rate of $\sim 99 \%$, and the cancerous spectra, a rate of $\sim 96 \%$. As the class imbalance grows, spectra in the minority class are randomly duplicated to ensure the numbers are the same in each class. The difference between this and under-sampling is that all the data from the majority class are unique, while those from the minority class will contain duplicates. However, under-sampling reduces the total number of spectra to twice the number in the minority class, while over-sampling increases the total number to twice that of the majority class. Therefore, in this case the 90:10 training set contains 4500 unique cancer spectra, but only 500 unique NAT spectra, the additional 4000 NAT spectra being randomly selected duplicates.

\section{Random Forests results}

Previous studies have employed the Random Forests algorithm to explore classification of cancerous and normal-associated tissues. For comparison purposes, we took the same test and training sets, generated above, and constructed a range of models using the Random Forests approach. The performance and outcomes were then assessed.

The Random Forests algorithm incorporated into the MATLAB Statistics and Machine Learning Toolbox was used (fitcensemble.m, with the appropriate parameters). 500 trees were used to train the classifier. The minimum node size to split was left at the default value of one.

Same overall training set size, unbalanced class sizes, unique spectra. With unbalanced training data comprising the same total number of spectra, Training sets A above, the outcome again begins with a similar classification rate of approximately $94 \%$ for each class. However, as shown in Fig. 11, as the imbalance in the data increases, it is the cancerous spectra that exhibit improved classification accuracy, while the normal-associated tissue class drops in accuracy to a mean of $\sim 76 \%$ with increased standard deviation. This indicates that Random Forests has difficulty in managing heavily unbalanced classes.

Different overall training set size, balanced class sizes (under-sampled), unique spectra. Fig. 12 shows the percentage of test data correctly classified for the Random Forests algorithm when trained using balanced data sets containing unique spectra: Training sets B. The 50:50 training data set, containing 2500 spectra of cancerous tissue and 2500 spectra

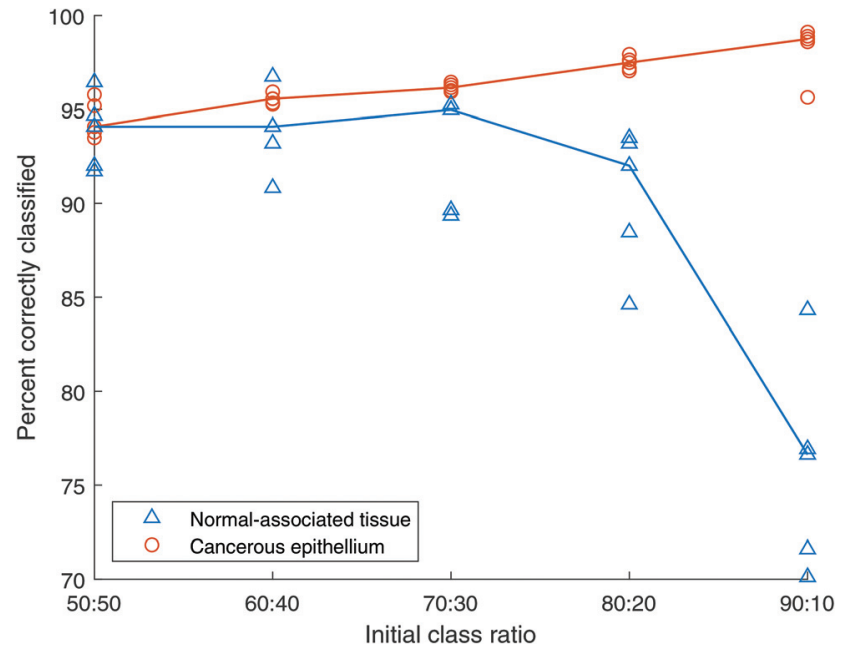

Fig. 11 Classification accuracy of Random Forests with unbalanced training sets A. All spectra are unique. Blue triangles show normalassociated tissue (NAT) results, orange circles show cancer tissue results. The lines represent the median of NAT repeats in blue and cancer repeats in orange.

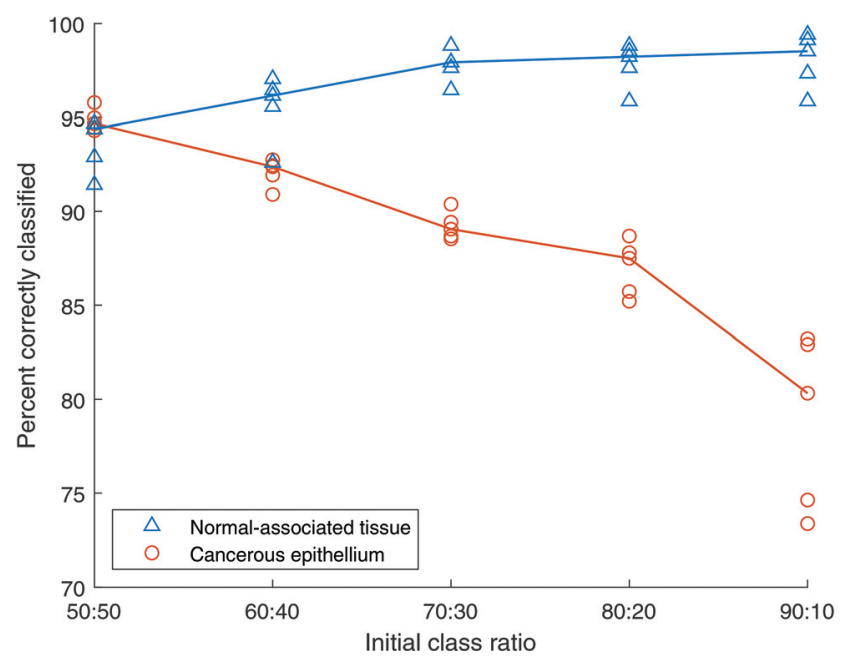

Fig. 12 Random Forests classification accuracy using training sets B, with balanced classes of decreasing size, generated by under-sampling. All spectra are unique. Blue triangles show normal-associated tissue (NAT) results, orange circles show cancer tissue results. The lines represent the median of NAT repeats in blue and cancer repeats in orange.

of normal-associated tissue, indicates a similar classification accuracy of approximately $94 \%$ for each class type, with the cancer spectra showing a slightly smaller standard deviation. As the total number of spectra in these balanced training sets decreases to 1000 , the classification of normal-associated tissue improves, while that of cancerous tissue decreases. The standard deviation of classification rate for the cancer spectra also increases. This could be due to the limited number of spectra in the highly under-sampled cases leading the algorithm to construct overfitted models. 


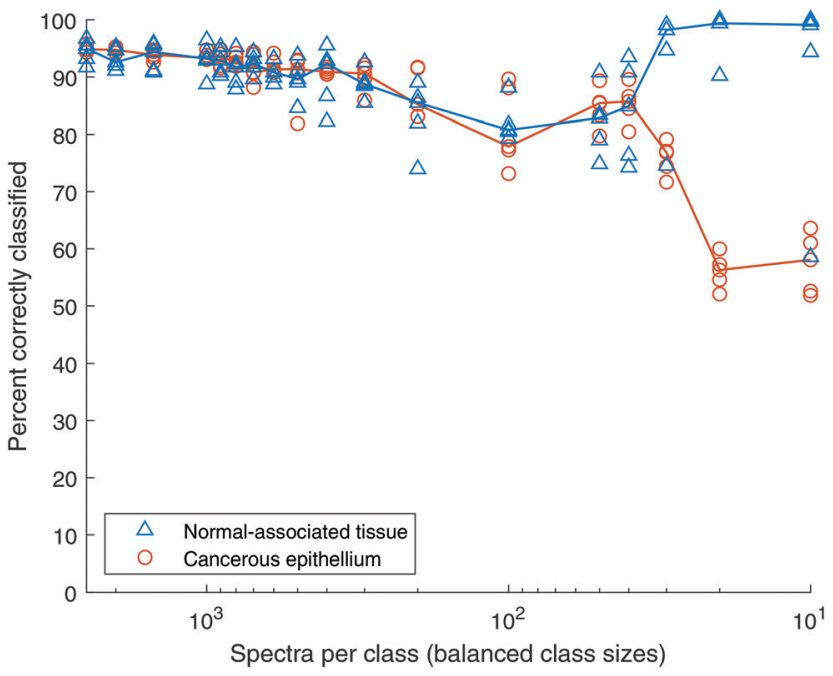

Fig. 13 Classification accuracy of Random Forests with balanced classes of decreasing overall size. All spectra are unique Blue triangles show normal-associated tissue (NAT) results, orange circles show cancer tissue results. The lines represent the median of NAT repeats in blue and cancer repeats in orange.

When the total number of spectra is further reduced, the Random Forests classifier retains a level of performance over $70 \%$ until there are only 50 spectra of each class in the training data, as shown in Fig. 13. Note that in contrast to AdaBoost, the model does not fail completely. However, with the NAT spectra indicating approximately $100 \%$ accuracy, the cancer spectra fall to $\sim 50 \%$, which is the equivalent of random chance in a two-class model.

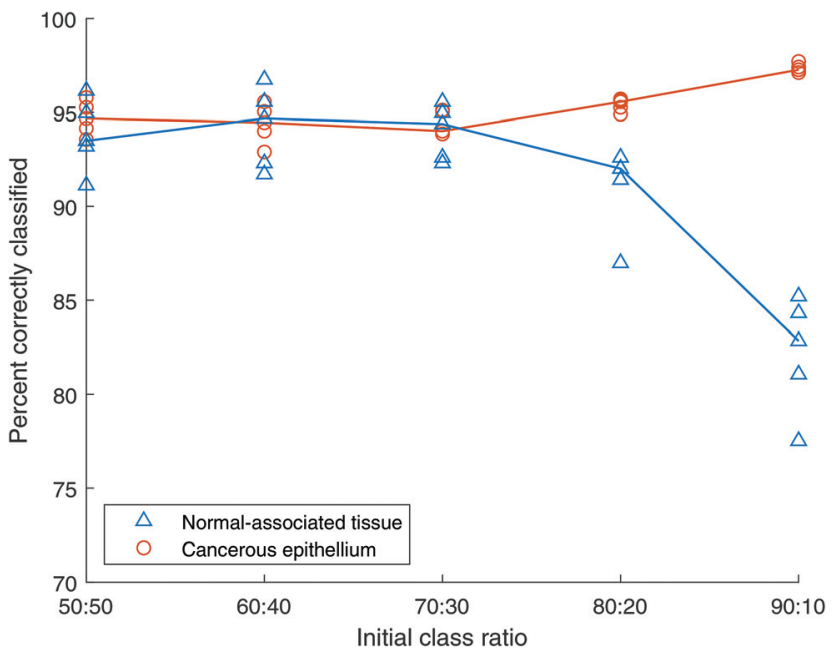

Fig. 14 Random Forests classification accuracy with different unbalanced class ratios being balanced using the over-sampling approach. Training sets $C$, where the minority class contains duplicates. Blue triangles show normal-associated tissue (NAT) results, orange circles show cancer tissue results. The lines represent the median of NAT repeats in blue and cancer repeats in orange.
Different overall training set size, balanced class sizes (oversampled), minority class duplicated. In the scenario where the minority class is over-sampled to produce balanced class sizes, Training sets $\mathrm{C}$ above, the Random Forests algorithm produces an outcome similar to that from unbalanced data, as shown in Fig. 14. The correct classification rate is almost equal for the two classes until a ratio of $70: 30$ is reached, whereupon the majority cancer class accuracy increases, and the minority normal-associated class falls. Again, as seen with AdaBoost, the over-sampled data behaves in a similar fashion to the unbalanced data shown in Fig. 11.

\section{Discussion}

The first observation is that with 2000 or more spectra, we can correctly classify over $90 \%$ of the spectra, regardless of the sampling method, or algorithm employed. This indicates that infrared spectroscopy is a useful tool for the detection of cancerous tissue, in the presence of normal-associated tissue, in breast cancer diagnosis.

The models constructed from unbalanced data using AdaBoost showed good consistency across a wide range of class imbalance. Therefore, this study would suggest that there is no need to perform re-sampling of data, prior to analysis, when using this algorithm; with the proviso that sufficient training examples are available.

Under-sampling involves the removal of data from the majority class, which is likely to have the effect of constructing a less accurate model as the total number of spectra decreases. This can be seen in Fig. 8 and 9 for AdaBoost, and Fig. 12 and 13 for Random Forests. Limited training data prevents the derived models from learning the breadth of variability required to correctly predict the test data, and therefore any previously unseen data in operation.

Both AdaBoost and Random Forests showed similar trends in classification accuracy when comparing unbalanced data with its over-sampled version. With increased imbalance there is a large duplication of examples in the minority class. Therefore, although there appear to be sufficient spectra in the minority class, its variability is low. When the algorithm attempts to learn from these data it is presented with a majority class containing wide variability, capable of capturing the entire space of that tissue type, but a minority class with insufficient variability relating to its tissue type. The model overfits the minority class which then performs poorly when attempting to predict a range of test data. This could explain the difference in standard deviation of the cancer (majority) and NAT (minority) repeated examples, at 90:10 initial class ratio, in Fig. 10 and 14.

Over-sampling also increases the total number of spectra that the algorithms must manage, due to the replication of spectra in the minority class, thereby increasing the compute resource and analysis time required. 


\section{Comparison between AdaBoost and Random Forests} approaches

When comparing AdaBoost with Random Forests, it is clear from Fig. 7 and 11 that AdaBoost is the more robust method when presented with unbalanced data. However, both algorithms require over 100 spectra of each class to perform with greater than $80 \%$ classification accuracy. With modern infrared imaging instrumentation this level of data is easily acquired, but the tissue samples must contain sufficient cancer cells in the sampled region to develop a useful model.

AdaBoost is an iterative algorithm and so both the model building exercise, and unseen data prediction, are linear. Random Forests generates many decision trees, each independent. Therefore, Random Forests is amenable to parallel processing on modern computer processors, thus speeding up both model building and predictive analysis.

\section{Conclusions}

Both AdaBoost and Random Forests algorithms have been shown to give excellent classification performance, on the order of 95\% accuracy, in separating infrared spectra of cancerous epithelium tissue from normal-associated tissue on the tissue microarray used in this study. Further work is required to determine whether this is a typical result when assessing model transfer across samples, instruments, and laboratories.

AdaBoost is shown to be a robust algorithm in the presence of data of unbalanced composition, out-performing Random Forests at larger degrees of imbalance.

Given the stability of the AdaBoost algorithm on unbalanced data we suggest that the re-sampling approaches discussed in this paper may not be required.

\section{Author contributions}

Jiayi Tang: conceptualisation, data curation (lead), formal analysis (lead), investigation (lead), methodology (lead), software (lead), validation (lead), visualisation, writing the original draft (lead), review \& editing. Alex Henderson: data curation, formal analysis, software, validation, review \& editing. Peter Gardner: conceptualisation, project administration (lead), resources (lead), supervision (lead), visualisation, review \& editing.

\section{Conflicts of interest}

There are no conflicts to declare.

\section{Acknowledgements}

The authors thank Daniela Kurfürstová of the Palacký University Olomouc, Czech Republic, for helpful discussion relating to cancer pathology. We also thank the Williamson
Trust for generous support towards the purchase of the benchtop FTIR microscope used in this study.

\section{References}

1 D. C. Fernandez, R. Bhargava, S. M. Hewitt and I. W. Levin, Nat. Biotechnol., 2005, 23, 469-474.

2 E. Gazi, M. Baker, J. Dwyer, N. P. Lockyer, P. Gardner, J. H. Shanks, R. S. Reeve, C. A. Hart, N. W. Clarke and M. D. Brown, Eur. Urol., 2006, 50, 750-761.

3 M. J. Baker, E. Gazi, M. D. Brown, J. H. Shanks, N. W. Clarke and P. Gardner, J. Biophotonics, 2009, 2, 104113.

4 M. J. Pilling, A. Henderson, J. H. Shanks, M. D. Brown, N. W. Clarke and P. Gardner, Analyst, 2017, 142, 1258-1268.

5 F. Großerueschkamp, A. Kallenbach-Thieltges, T. Behrens, T. Brüning, M. Altmayer, G. Stamatis, D. Theegarten and K. Gerwert, Analyst, 2015, 140, 2114-2120.

6 X. Mu, M. Kon, A. Ergin, S. Remiszewski, A. Akalin, C. M. Thompson and M. Diem, Analyst, 2015, 140, 24492464.

7 A. Kallenbach-Thieltges, F. Großerüschkamp, A. Mosig, M. Diem, A. Tannapfel and K. Gerwert, J. Biophotonics, 2013, 6, 88-100.

8 N. Kröger-Lui, N. Gretz, K. Haase, B. Kränzlin, S. Neudecker, A. Pucci, A. Regenscheit, A. Schönhals and W. Petrich, Analyst, 2015, 140, 2086-2092.

9 C. L. Song, M. Z. Vardaki, R. D. Goldin and S. G. Kazarian, Anal. Bioanal. Chem., 2019, 411, 6969-6981.

10 C. Kuepper, F. Großerueschkamp, A. Kallenbach-Thieltges, A. Mosig, A. Tannapfel and K. Gerwert, Faraday Discuss., 2016, 187, 105-118.

11 C. Hughes, J. Iqbal-Wahid, M. Brown, J. H. Shanks, A. Eustace, H. Denley, P. J. Hoskin, C. West, N. W. Clarke and P. Gardner, J. Biophotonics, 2013, 6, 73-87.

12 M. Sattlecker, N. Stone and C. Bessant, TrAC, Trends Anal. Chem., 2014, 59, 17-25.

13 N. I. R. Yassin, S. Omran, E. M. F. El Houby and H. Allam, Comput Methods Programs Biomed, 2018, 156, 25-45.

14 H. Fabian, N. A. N. Thi, M. Eiden, P. Lasch, J. Schmitt and D. Naumann, Biochim. Biophys. Acta, Biomembr., 2006, 1758, 874-882.

15 D. M. Mayerich, M. Walsh, A. Kadjacsy-Balla, S. Mittal and R. Bhargava, in Progress in Biomedical Optics and Imaging Proceedings of SPIE, ed. M. N. Gurcan and A. Madabhushi, 2014, p. 904107.

16 P. Bassan, M. J. Weida, J. Rowlette and P. Gardner, Analyst, 2014, 139, 3856-3859.

17 S. Mittal, T. P. Wrobel, L. S. Leslie, A. Kadjacsy-Balla and R. Bhargava, in Medical Imaging 2016: Digital Pathology, ed. M. N. Gurcan and A. Madabhushi, 2016, p. 979118.

18 J. Tang, D. Kurfürstová and P. Gardner, Clin. Spectrosc., 2021, 3, 100008.

19 S. Mittal, C. Stoean, A. Kajdacsy-Balla and R. Bhargava, Front. Bioeng. Biotechnol., 2019, 7, 246. 
20 J. Bell, Machine Learning: Hands-On for Developers and Technical Professionals, John Wiley \& Sons, Inc, Indianapolis, IN, USA, 2014.

21 K. P. Murphy, Machine Learning a Probabilistic Perspective, MIT Press, Cambridge, Massachusetts, Illustrate., 2012.

22 L. S. Leslie, T. P. Wrobel, D. Mayerich, S. Bindra, R. Emmadi and R. Bhargava, PLoS One, 2015, 10, e0127238.

23 S. Mittal, K. Yeh, L. S. Leslie, S. Kenkel, A. Kajdacsy-Balla and R. Bhargava, Proc. Natl. Acad. Sci. U. S. A., 2018, 115, E5651-E5660.

24 T. G. Dietterich, in First International Workshop, MCS 2000 Cagliari, Italy, June 21-23, 2000 Proceedings, 2000, vol. 1857, pp. 1-15.

25 A. Cutler, D. R. Cutler and J. R. Stevens, in Ensemble Machine Learning, Springer US, Boston, MA, 2012, pp. 157-175.

26 Y. Freund and R. E. Schapire, in Proceedings of the 13th International Conference on Machine Learning, Morgan Kaufmann Publishers, Burlington, Massachusetts, California, 1996, pp. 208-219.

27 Y. Freund, in Proceedings of the twelfth annual conference on Computational learning theory - COLT '99, ACM Press, New York, New York, USA, 1999, pp. 102-113.

28 R. E. Schapire, in Empirical Inference, Springer Berlin Heidelberg, Berlin, Heidelberg, 2013, pp. 37-52.

29 A. J. Ferreira and M. A. T. Figueiredo, in Ensemble Machine Learning, Springer US, Boston, MA, 2012, pp. 35-85.
30 C. Seiffert, T. M. Khoshgoftaar, J. Van Hulse and A. Napolitano, IEEE Trans. Syst., Man, Cybern. A, Syst. Humans, 2010, 40, 185-197.

$31 \mathrm{~N}$. Japkowicz, in AAAI Workshop on Learning from Imbalanced Data Sets, 2000, pp. 10-15.

32 E. El-shafeiy and A. Abohany, in Advances in Intelligent Systems and Computing, Springer, 2020, vol. 1153 AISC, pp. 81-91.

33 A. Ali, S. M. Shamsuddin and A. L. Ralescu, Int. J. Adv. Soft Comput. Appl., 2015, 7, 176-204.

34 H. He and E. A. Garcia, IEEE Trans. Knowl. Data Eng., 2009, 21, 1263-1284.

35 P. Branco, L. Torgo and R. P. Ribeiro, ACM Comput. Surv., 2016, 49, 1-50.

36 M. M. Rahman and D. N. Davis, Int. J. Mach. Learn. Comput., 2013, 224-228.

37 G. M. Weiss, ACM SIGKDD Explor. Newsl., 2004, 6, 7-19.

38 F. Lyng, E. Gazi and P. Gardner, in RSC Analytical Spectroscopy Series, ed. D. Moss, Royal Society of Chemistry, Cambridge, 2010, pp. 147-191.

39 D. Aran, R. Camarda, J. Odegaard, H. Paik, B. Oskotsky, G. Krings, A. Goga, M. Sirota and A. J. Butte, Nat. Commun., 2017, 8, 1077.

40 S. R. Lakhani, International Agency for Research on Cancer and World Health Organization, in WHO Classification of Tumours of the Breast, International Agency for Research on Cancer, Lyon, France, 4th edn, 2012.

41 R. Blagus and L. Lusa, BMC Bioinformatics, 2013, 14, 106. 